\title{
Diagnostic accuracy of high-resolution attenuation-corrected Anger-camera SPECT in the detection of coronary artery disease
}

\author{
Harshal R. Patil, MD, ${ }^{\mathrm{a}, \mathrm{b}, \mathrm{d}}$ Timothy M. Bateman, $M D,{ }^{\mathrm{a}, \mathrm{b}, \mathrm{c}, \mathrm{d}}$ A. Iain McGhie, $M D,{ }^{\mathrm{a}, \mathrm{b}}$ \\ Eric V. Burgett, MS, CNMT, ${ }^{\text {a }}$ Staci A. Courter, MA, ${ }^{c}$ James A. Case, PhD, ${ }^{\mathrm{c}, \mathrm{d}}$ and \\ Gary V. Heller, MD, PhD
}

Background. There is limited data on diagnostic accuracy of recently introduced highresolution Anger (HRA) SPECT incorporating attenuation correction (AC), noise reduction, and resolution recovery algorithms. We therefore studied 54 consecutive patients (excluding those with prior MI or cardiomyopathy) who had HRA-AC SPECT and coronary angiography (CA) $\leq 30$ days and no change in symptoms.

Methods. The HRA-AC studies were acquired in $128 \times 128$ matrix $(3.2 \mathrm{~mm}$ pixel) format with simultaneous Gd-153 line-source AC. Measured variables were image quality, interpretive certainty, sensitivity and specificity for any CAD, sensitivity for single- and multivessel CAD, and the influence of gender, body mass index (BMI), and stress modality.

Results. The mean age of the patients was $66 \pm 11$ years with a BMI of $32 \pm 7 \mathrm{~kg} \cdot \mathrm{m}^{-2}$. Mean interpretive certainty score was 2.7 on a 3-point scale and mean image quality score was 3.3 on a 4-point scale. Stress perfusion defects were detected in 34 of 38 patients with obstructive CAD [sensitivity 89\%, 95\% confidence interval (CI) 76\% -95\%]. The specificity was 75\% (CI 51\% -90\%) and overall diagnostic accuracy was 85\% (CI 73\%-92\%). Accuracy did not differ for females vs males, for BMI $\leq 30$ vs $>30$, or for pharmacologic vs exercise SPECT. Sensitivity for single-vessel disease was $88 \%$ (CI 69\%-96\%) and for multivessel disease was 93\% (CI 69\%-99\%).

Conclusion. New Anger technology incorporating innovative improvements results in high image quality with excellent interpretive certainty and high diagnostic accuracy. (J Nucl Cardiol 2014;21:127-34.)

Key Words: Single photon emission computed tomography $\bullet$ myocardial perfusion imaging • attenuation correction $\cdot$ resolution recovery

\section{INTRODUCTION}

Single photon emission computed tomography (SPECT) for myocardial perfusion imaging (MPI) is widely

From the Saint-Luke s Mid America Heart Institute, ${ }^{a}$ Kansas City, MO; School of Medicine, ${ }^{\mathrm{b}}$ University of Missouri-Kansas City, Kansas City, MO; Cardiovascular Imaging Technologies, ${ }^{\mathrm{c}}$ Kansas City, MO; ASPIRE Foundation, ${ }^{\mathrm{d}}$ Kansas City, MO; and Hartford Hospital, ${ }^{\mathrm{e}}$ Hartford, CT

Received for publication Apr 10, 2013; final revision accepted Oct 30, 2013.

Reprint requests: Timothy M. Bateman, MD, Saint-Luke's Mid America Heart Institute, 4330 Wornall Road, Suite 2000, Kansas City, MO 64111; tbateman@saint-lukes.org.

$1071-3581 / \$ 34.00$

Copyright (c) 2013 American Society of Nuclear Cardiology.

doi:10.1007/s12350-013-9817-9 used to guide medical decisions such as need for cardiac catheterization in patients with suspected coronary artery disease $(\mathrm{CAD})$, need for revascularization, establishing prognosis, assessing the effectiveness of therapy, and evaluating viability among those with left ventricular dysfunction. ${ }^{1}$ In 2009, approximately 9.3 million patients underwent SPECT MPI in the United States alone. ${ }^{2}$ The usefulness of SPECT is highly dependent on its quality, which in turn reflects among other variables its count density, configuration of the system used (detectors, collimators, gantry geometry), and reconstruction methods. ${ }^{3}$

Recently, a variety of reconstruction methods variously incorporating line-source or computed tomography-based attenuation correction $(\mathrm{AC}),{ }^{4,5}$ collimator resolution recovery (RR), ${ }^{6}$ scatter correction, noise control, iterative reconstruction, and detector distance optimization have been proposed to overcome 
limitations of traditional FBP SPECT MPI, and to address increasing competition from myocardial perfusion positron emission tomography (PET) and cadmium zink telluride SPECT. Several publications have demonstrated that these advances can retain image quality and accuracy at shorter acquisition times ${ }^{7}$ or using lower tracer dosages. ${ }^{8}$ We previously investigated one of these reconstruction algorithms and showed improved image quality when compared to FBP using both full-time and half-time data with no loss in sensitivity and a significant improvement in normalcy and specificity. ${ }^{6}$ Furthermore, half-time stress-only attenuation-corrected SPECT can achieve results equivalent to conventional rest/stress scanning and significantly improve operational efficiency without sacrificing accuracy. ${ }^{9}$

In this study, we extend our work with new acquisition and processing algorithms by evaluating SPECT myocardial perfusion images acquired into a 128 by 128 matrix (3.2 $\mathrm{mm}$ pixel sizes), along with iterative reconstruction, resolution recovery, noise reduction, and line-source AC (Astonish-128, Philips Medical Systems, Milpitas, CA). The primary aim of this study was to evaluate through a blinded read the diagnostic accuracy of high-resolution attenuation-corrected Anger (HRAAC) SPECT for detection of CAD in a consecutive cohort of patients who were referred to coronary angiography (CA) on the basis of their traditionally performed SPECT studies (64 by 64 matrix with filtered back projection and line-source $\mathrm{AC}$ ).

\section{METHODS}

\section{Study Design}

This study was designed to evaluate interpretive certainty and diagnostic accuracy of images acquired in $128 \times 128(3.2 \mathrm{~mm}$ pixel) formats with simultaneous Gd153 line-source $\mathrm{AC}$ and processed using the previously published full-time Astonish (FTA) algorithm. ${ }^{6,13}$ The accuracy gold standard was $\mathrm{CA}$ that had been performed within 30 days and no change in symptom status in between. A consensus-blinded interpretation of HRA-AC rest-stress or stress-only SPECT images of patients without prior myocardial infarction (MI) or left ventricular dysfunction was performed in batch format by 2-reader consensus. Reversible perfusion defects were correlated with $\mathrm{CA}$. The presence or absence of $\mathrm{CAD}$ was determined from the clinical CA reports, with luminal diameter narrowing of the three main coronary arteries or their major branches of $\geq 70 \%$ or of the left main coronary artery of $\geq 50 \%$ being used as the criteria for significance. Measured variables were image quality, interpretive certainty, sensitivity and specificity for obstructive CAD, and sensitivity for detection of single- and multivessel CAD. The influence of gender, body mass index (BMI), and stress modality on accuracy were also assessed.

\section{Patients}

Nuclear cardiology lab data were reviewed to identify all patients $(\mathrm{n}=72)$ between December 2010 and January 2012 who underwent vasodilator, exercise, or combined exercisevasodilator HRA-SPECT acquired in $128 \times 128$ format with $\mathrm{AC}$, and who underwent $\mathrm{CA}$ within 30 days with no change in clinical status. Of 72 patients, 18 were excluded based on predefined criteria: (1) prior MI; (2) BMI greater than $50 \mathrm{~kg} \cdot \mathrm{m}^{-2}$; (3) left ventricular ejection fraction (LVEF) of $<40 \%$ based on their SPECT study because of the known impact of cardiomyopathy on myocardial perfusion independent of epicardial $\mathrm{CAD}$; and (4) prior cardiac transplant. The remaining 54 patients were included for this study.

Thirty-nine $(73 \%)$ of the patients underwent adenosine or regadenoson vasodilator stress and $15(27 \%)$ underwent treadmill exercise stress. All stress testing was performed in accordance with ASNC procedural guidelines. ${ }^{3}$ The SaintLuke's Hospital Institutional Review Board approved this study.

\section{Image Acquisitions}

Studies were acquired in $128 \times 128$ (3.2 mm pixel) format with simultaneous Gd-153 line-source AC (CardioMD, Philips, Milpitas, CA). Data were then compressed into $64 \times 64(6.4 \mathrm{~mm})$ pixel format for clinical interpretations. The 128 matrix data were not used for clinical interpretations. In accordance with ASNC imaging guidelines ${ }^{3}$ data were acquired over 64 projections at 40 seconds per projection for the stress and 35 seconds per projection for the rest images, using a $180^{\circ}$ RAO-LPO orbit beginning 15-45 minutes after 11-40 $\mathrm{mCi}$ of Tc-99m sestamibi. The collimators were lowenergy high resolution, and the energy windows were set at $140 \mathrm{keV} \pm 10 \%$ for the emission and $100 \mathrm{keV} \pm 10 \%$ for the Gd-153 transmission data. An additional $118 \mathrm{keV} \pm 6 \%$ photo peak window was used to compensate for down scatter of Tc$99 \mathrm{~m}$ into the Gd-153 energy window. ${ }^{3,10,11}$ All data were acquired using 16-frame ECG-gating.

\section{Image Processing}

Imaging data were processed using Astonish-128 with $\mathrm{AC}^{12,13}$ (VantagePro, Philips, Milpitas, CA) applied to both the perfusion images and each of the 16 frames of the stress and rest gated images. The transverse perfusion images were reconstructed with the OSEM-based Astonish algorithm, as recommended by the manufacturer, using four iterations and eight subsets and a Hanning match filter of cutoff 0.75 . The ECG-gated images were reconstructed using four iterations, eight subsets, and a Hanning match filter of cutoff 0.4. The transmission maps for the 64-projection studies were reconstructed using the previously validated method for 64 projections. ${ }^{14}$ For all attenuation map reconstructions, a uniform initial estimate and 30 iterations of a Bayesian algorithm ${ }^{14}$ applied to the downscatter-compensated projection data were used. Truncation compensation for artifacts occurring in the small field of view transmission images ${ }^{4}$ was applied to all images. When applying AC, Astonish 
incorporates an attenuation map-based photopeak scatter correction algorithm in addition to the resolution recovery. ${ }^{15}$

\section{Image Interpretation and Scoring}

Two experienced nuclear cardiologists, blinded to all clinical information and to the results of $\mathrm{CA}$, reviewed processed emission, ECG-gated, and rotating projection images. Perfusion and gated image quality was interpreted using a 4-point scale of 1 (low) to 4 (excellent). Studies were categorized as definitely normal, probably normal, equivocal, probably abnormal, and definitely abnormal. Interpretive certainty was based on image categorizations as definitely normal or abnormal (score of 3 ), probably normal or abnormal (score of 2 ), or equivocal (score of 1). Perfusion was graded on a 5 -point scale $(0=$ normal, $1=$ mildly reduced perfusion, $2=$ moderately reduced perfusion, $3=$ severely reduced perfusion, and $4=$ absent perfusion) using the standard 17 -segment model. ${ }^{16}$ The visually interpreted segmental scores for each imaging study were added to derive a summed stress score (SSS), a summed rest score (SRS), and a summed difference score (SDS).

\section{Coronary Angiography}

All patients underwent $\mathrm{CA}$ using standard techniques within 30 days of their SPECT scan. Obstructive CAD was derived from the angiographic reports; angiographic images were not re-read for this study. The angiographic criterion used to define the presence of significant obstructive CAD was a visually determined diameter stenosis of $\geq 70 \%$ for the left anterior descending, left circumflex, and right coronary arteries or their major branches, and $\geq 50 \%$ for the left main coronary artery.

\section{Statistical Analysis}

In comparisons of patients within groups, categorical variables were calculated as percentages and then compared by use of a Chi square $\left(\chi^{2}\right)$ test. ${ }^{17}$ The ordinal variables were compared using $t$ test. Diagnostic accuracy for CAD was determined with definitely or probably normal or abnormal being correct responses; a positive diagnosis was defined as a study that was probably or definitely abnormal, and a negative diagnosis was defined as a study that was probably or definitely normal. Sensitivity, specificity, and accuracy were calculated within the angiography group and compared at a cutoff disease severity of $\geq 70 \%$ luminal diameter narrowing. $95 \%$ confidence intervals (CIs) for proportions were calculated by Wilson procedure without correction for continuity. ${ }^{18}$ The individual coronary territories were compared in the same manner. Statistical significance was denoted by $P$ values $<.05$.

\section{RESULTS}

\section{Demographics}

The average age of the 54 studied patients was $66 \pm 11$ years and $74 \%(n=40)$ were male. The
Table 1. Baseline characteristics of the 54 patients in this study

\begin{tabular}{ll}
\hline Male 40 & $(74 \%)$ \\
Age, years (mean \pm SD) & $66 \pm 10.9$ \\
Body mass index (mean \pm SD) & $31.5 \pm 6.53$ \\
Hypertension & $42(77 \%)$ \\
Hypercholesterolemia & $45(83 \%)$ \\
Smoking & $12(22 \%)$ \\
Family history for CAD & $18(33 \%)$ \\
Diabetes mellitus & $21(39 \%)$ \\
Prior known CAD & $29(54 \%)$ \\
Prior PCI & $20(37 \%)$ \\
Exercise stress & $15(27 \%)$ \\
Vasodilator stress & $39(73 \%)$ \\
Tc-99m sestamibi activity (mean \pm SD) \\
Rest & $13.5 \pm 8.2 \mathrm{mCi}$ \\
Stress & $30.6 \pm 6.4 \mathrm{mCi}$ \\
Ejection Fraction (mean \pm SD) & \\
Rest & $57.6 \% \pm 12 \%$ \\
Stress & $57 \% \pm 11.3 \%$ \\
Coronary angiography results $(70 \%$ threshold) \\
0-vessel & $16(30 \%)$ \\
Single-vessel CAD & $24(44 \%)$ \\
Multivessel CAD & $14(26 \%)$ \\
2-vessel CAD & $10(19 \%)$ \\
3-vessel CAD & $4(7 \%)$ \\
\hline
\end{tabular}

majority of the patients were overweight or obese: mean BMI was $31.5 \pm 6.5 \mathrm{~kg} \cdot \mathrm{m}^{-2}$ with a range of 18.1 $48 \mathrm{~kg} \cdot \mathrm{m}^{-2}$. Significant CAD was present in $70 \%$ $(\mathrm{n}=38): 44 \%(\mathrm{n}=24)$ patients had SVD and $26 \%$ $(\mathrm{n}=14) \quad$ had $\quad$ MVD $\quad(2 \mathrm{VD}=10 ; \quad 3 \mathrm{VD}=4) \quad($ see Table 1). The left anterior descending artery $(n=27)$ was the most commonly affected followed by the right coronary artery $(\mathrm{n}=19)$ and the left circumflex artery $(\mathrm{n}=8)$. The median time interval between SPECT and CA was 7.5 days, ranging from 0 to 27 days.

\section{Agreement Between HRA-AC SPECT and Coronary Angiography}

Overall detection of CAD. HRA-AC SPECT correctly detected 34 of 38 patients with obstructive CAD (sensitivity 89\%, 95\% CI 76\%-96\%). The sensitivity for detection of CAD in patients with SVD was $88 \%$ (95\% CI 69\%-96\%) and in patients with MVD was 93\% (95\% CI 69\%-99\%) (see Table 2). The sensitivity for overall CAD detection was equally high in men $(87 \%, 95 \%$ CI $91 \%-95 \%)$ and women $(100 \%, 95 \%$ CI $65 \%-100 \%)$, in the obese (BMI $\geq 30)$ (87\%, 95\% CI 68\%-95\%) and non-obese (93\%, 
Table 2. Detection of CAD in 54 patients who underwent coronary angiography

\begin{tabular}{lccc}
\hline & \multicolumn{3}{c}{ CAD by coronary angiography } \\
\cline { 2 - 4 } CAD by HRA-SPECT & No & Yes & Total \\
\hline No & 12 & 4 & 16 \\
Yes & 4 & 34 & 38 \\
Total & 16 & 38 & 54 \\
\hline
\end{tabular}

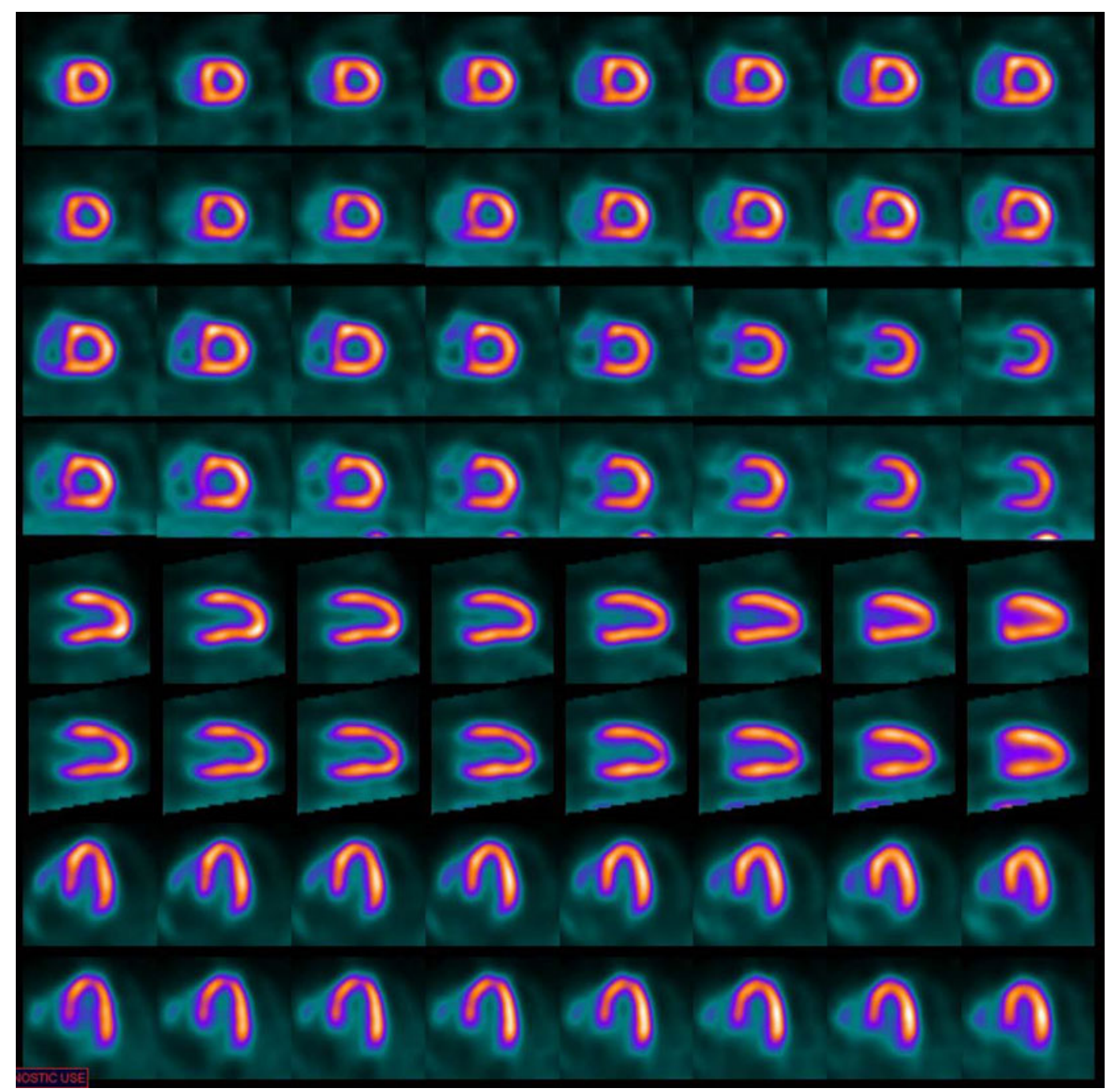

Figure 1. Normal rest/stress HRA-AC SPECT images in a 42-year-old woman with no prior history of CAD, symptoms of atypical angina, and numerous risk factors for CAD. Her BMI was $39.6 \mathrm{~kg} \cdot \mathrm{m}^{-2}$. She underwent an exercise stress test.

95\% CI 70\%-99\%), and in those tested with exercise $(88 \%, 95 \%$ CI $53 \%-98 \%)$ vs pharmacologically $(94 \%$, 95\% CI $80 \%-98 \%$ ). Figures 1, 2, and 3 show representative normal and abnormal images in women and men.
HRA-AC SPECT correctly identified the absence of CAD in 12 of 16 patients who had no significant CAD on their angiograms (see Table 2). The four false positive interpretations included two women and two men with BMI's of 25, 26, 32, and 45. Three of the four underwent 


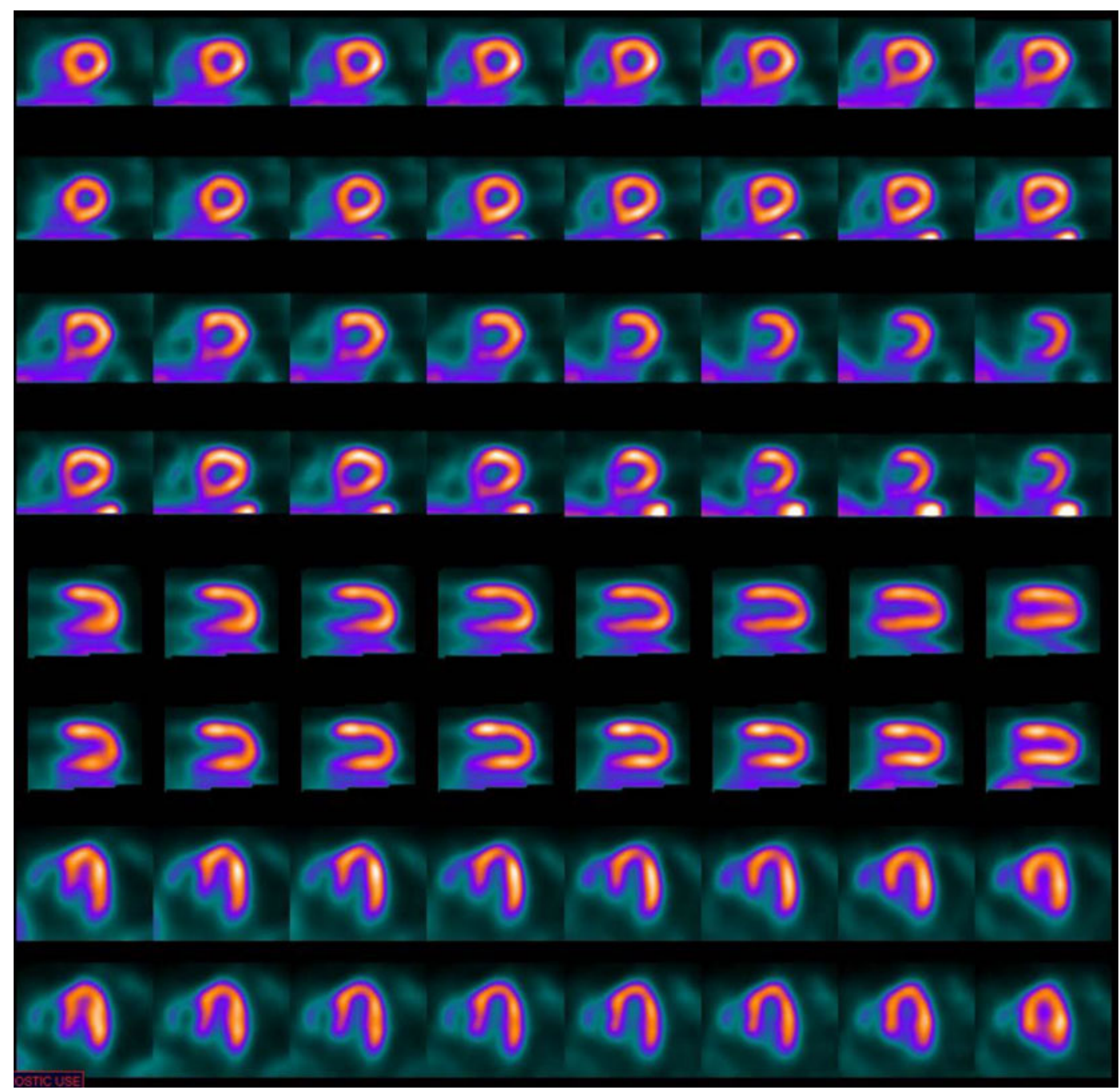

Figure 2. Abnormal rest/stress HRA-AC SPECT images in a 73-year-old man with BMI $30 \mathrm{~kg} \cdot \mathrm{m}^{-2}$ with bilateral shoulder pain and dyspnea. He underwent a symptom-limited combined exercise/regadenoson stress test. Coronary angiography showed a $100 \%$ stenotic lesion in the RCA, a $40 \%$ stenosis in the LAD, and a $30 \%$ stenosis in the LCx.

pharmacologic stress. In all four, the HRA-SPECT was interpreted as showing single-vessel CAD-LAD in two and RCA in two. All four had CAD by cath: scored $<40 \%$ in two, and $<25 \%$ in the other two.

Relation between perfusion defects and angiographic extent of CAD. While HRA-AC SPECT was interpreted as ischemic in $88 \%(21 / 24$ patients) with SVD, only 19 of the 24 were correctly identified as having only SVD (see Table 3). Three were classified as normal and two as having MVD. Of the 14 patients with MVD, SPECT was abnormal in 13 (93\%), but was interpreted as showing MVD in only 6 (43\%). The incorrect diagnoses included one patient that showed no abnormalities and seven that were interpreted as having SVD. There was no statistically significant difference in the summed perfusion scores for SVD and MVD. The average SSS for patients with SV CAD was $6.9( \pm 4.4)$ and for MVD was $7.8( \pm 4.7)(P=.19)$.

\section{Image Quality and Interpretive Certainty}

The mean image quality score for rest and poststress perfusion images was 3.3 and 3.2, respectively, on the 4-point scale. $86 \%$ of the rest images and $80 \%$ of the stress images were scored either 3 or 4 on the 4-point scale.

The mean interpretive certainty score was 2.7 on the 3-point scale. $72 \%(\mathrm{n}=39)$ of the studies were interpreted as definitely normal or definitely abnormal; none were scored equivocal. 


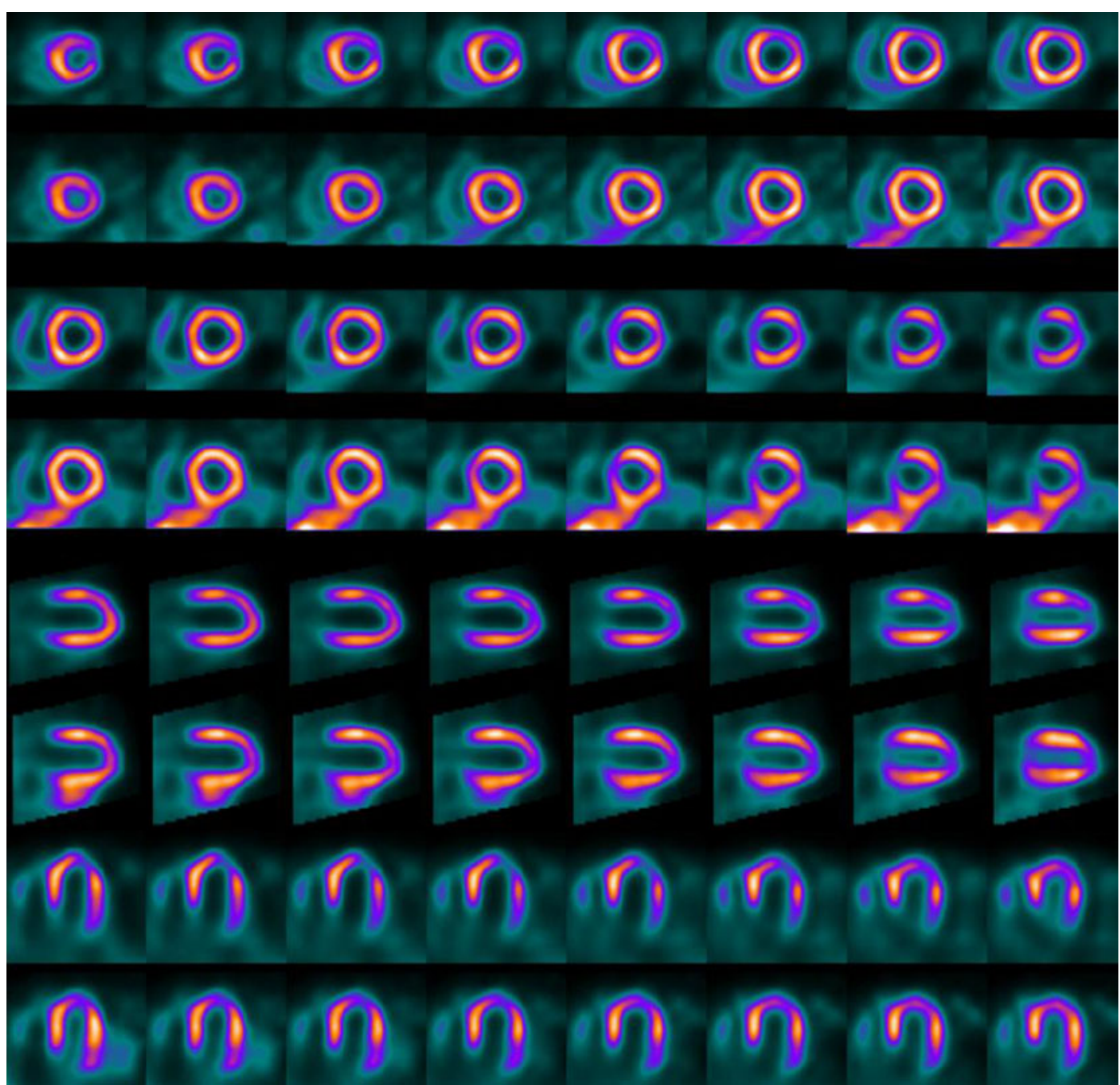

Figure 3. Abnormal rest/stress HRA-AC SPECT images in a 39-year-old man with exerciseinduced chest pain. Coronary angiography revealed high-grade diagonal and ramus intermediate stenoses.

Table 3. Extent of CAD in 38 patients with obstructive disease at coronary angiography

\begin{tabular}{lrrrr}
\hline & \multicolumn{4}{c}{ \# Diseased coronaries by angiography } \\
\cline { 2 - 5 } \# Diseased vessels by HRA-SPECT & $\mathbf{0}$ & SVD & MVD & Total \\
\hline 0 & 12 & 3 & 1 & 16 \\
SVD & 3 & 19 & 7 & 29 \\
MVD & 1 & 2 & 6 & 9 \\
Total & 16 & 24 & 14 & 54 \\
\hline
\end{tabular}

\section{DISCUSSION}

Our study evaluated image quality, interpretive certainty, and diagnostic performance of one of the newly introduced software packages designed to enhance quality of SPECT images acquired using traditional Anger instrumentation. The Astonish software we evaluated (Philips Medical Systems, Milpitas, $\mathrm{CA}$ ) incorporated $128 \times 128$ matrix acquisition, AC, scatter correction, iterative reconstruction, adaptive noise suppression, and measured resolution recovery of 
counts. ${ }^{6,13}$ The resulting images are characterized by higher spatial and contrast resolution compared against traditional reconstruction parameters, with the potential for both under and over-diagnosing CAD. The overall sensitivity for diagnosing obstructive CAD was $89 \%$, was equally high in men and in women, in those undergoing either exercise or pharmacologic stress, and in those with single as compared to multivessel CAD.

Sensitivity for overall recognition of obstructive CAD was $93 \%$ in those patients with multivessel CAD. However, correct classification of those patients with MVD was only $43 \%$. This is a well-known limitation of spatially relative interpretations of myocardial perfusion scans, and our results are similar to those reported in the past for both SPECT and PET. ${ }^{19}$ Our population was under-represented by 3-vessel disease, as only four patients had this.

Our diagnostic accuracy in this study in which the interpreters were blinded to all clinical information was superior to prior single- and multicenter studies using conventional attenuation-corrected SPECT, where sensitivity and specificity have ranged from $76 \%$ to $82 \%$ and $73 \%$ to $75 \%$, respectively. ${ }^{6,19-21}$

\section{Image Quality and Interpretive Certainty}

A major limitation of traditional SPECT MPI is image quality that is often compromised by attenuation artifacts, scatter, or adjacent "hot' structures that either touch or overlap portions of the myocardium. ${ }^{21,22}$ Less than optimal image quality affects interpretive certainty and diagnostic accuracy. ${ }^{23}$ In this study, HRA-AC SPECT achieved either good or excellent image quality in $80 \%-86 \%$ of all of the stress and the rest perfusion scans. The mean image quality score for rest and poststress images was 3.3 and 3.2, respectively, on the 4 -point scale. Interpretive certainty was high in $96 \%$ of the studies with average interpretive certainty score of 2.7 on the 3-point scale. Our study was not designed to determine the comparative contribution of the many potential influences on image quality built into the new software, but clearly the end-result was high quality images able to be interpreted with high certainty and high diagnostic accuracy.

\section{Study Limitations}

The major limitations of this study are its small sample size and that it reflects results in a single center. The results need verification from a larger number of patients derived from multiple centers. The AC was only derived from line-sources, which may not be extrapolatable to CT-based AC on the increasingly used hybrid SPECT/CT systems. Verification bias in which patients are referred to CA predominantly only after an abnormal scan may be a factor in this study, despite the fact that the clinical interpretations sent to referring physicians were based on traditional SPECT data. While our study demonstrates that these high-resolution SPECT images are interpretable in a manner that results in high diagnostic accuracy, the study was not designed or powered to determine comparative accuracy against traditional SPECT. The coronary angiograms were interpreted visually as opposed to quantitatively. This study did not evaluate quality or accuracy of function data.

\section{NEW KNOWLEDGE GAINED}

There is little published information about diagnostic accuracy of latest generation SPECT software that incorporates higher resolution, iterative reconstruction, noise reduction, measured resolution recovery, and attenuation correction. This interpretation study, blinded to all clinical information, establishes high diagnostic accuracy for detection of significant CAD, including single-vessel CAD.

\section{CONCLUSIONS}

The current study shows that high-resolution AC SPECT imaging provides high diagnostic sensitivity in obese and non-obese patients, in men and in women, with pharmacologic stress and exercise stress, and for detection of single-vessel CAD. As with traditional SPECT and Rb-82 PET, there remains significant underrecognition of presence of multivessel CAD.

\section{Disclosure}

This study was supported by a clinical research grant from Philips Medical Systems.

\section{References}

1. Klocke FJ, Baird MG, Lorell BH, Bateman TM, Messer JV, Berman DS, et al. ACC/AHA/ASNC guidelines for the clinical use of cardiac radionuclide imaging-executive summary: A report of the American College of Cardiology/American Heart Association Task Force on practice guidelines (ACC/AHA/ASNC Committee to revise the 1995 guidelines for the clinical use of cardiac radionuclide imaging). J Am Coll Cardiol 2003;42:1318-33.

2. Arlington Medical Resources (AMR) Data on File, 2009.

3. American Society of Nuclear Cardiology. Imaging guidelines for nuclear cardiology. J Nucl Cardiol 2006;13:e25-41. http://www. asnc.org/imageuploads/ImagingGuidelines.pdf.

4. Noble GL, Ahlberg AW, Kokkirala AR, Cullom SJ, Bateman TM, Cyr GM, et al. Validation of attenuation correction using transmission truncation compensation with a small field of view 
dedicated cardiac SPECT camera system. J Nucl Cardiol 2009; 16:222-32.

5. Hendel RC, Gibbons RJ, Bateman TM. Use of rotating (cine) planar projection images in the interpretation of a tomographic myocardial perfusion study. J Nucl Cardiol 1999;6:234-40.

6. Venero CV, Heller GV, Bateman TM, McGhie AI, Ahlberg AW, Katten D, et al. A multicenter evaluation of a new post-processing method with depth-dependent collimator resolution applied to fulltime and half-time acquisitions without and with simultaneously acquired attenuation correction. J Nucl Cardiol 2009;16:714-25.

7. Slomka PJ, Patton JA, Berman DS, Germano G. Advances in technical aspects of myocardial perfusion SPECT imaging. J Nucl Cardiol 2009;16:255-76.

8. Einstein AJ, Moser KW, Thompson RC, Cerqueira MD, Henzlova MJ. Radiation dose to patients from cardiac diagnostic imaging. Circulation 2007;116:1290-305.

9. Bateman TM, Heller GV, McGhie AI, Courter SA, Golub RA, Case JA, et al. Multicenter investigation comparing a highly efficient half-time stress-only attenuation correction approach against standard rest-stress Tc-99m SPECT imaging. J Nucl Cardiol 2009;16:726-35.

10. Case JA, Cullom SJ, Bateman TM. Myocardial perfusion SPECT attenuation correction. In: Iskandrian AE, Verani MS, editors. Nuclear cardiac imaging: Principles \& applications. 3rd ed. New York: Oxford University Press; 2002.

11. Henzlova MJ, Cerqueira MD, Mahmarian JJ, Yao SS. Stress protocols and tracers. J Nucl Cardiol 2006;13:e80-90.

12. Ye J, Song X, Zhao Z, Da Silva AJ, Wiener JS, Shao L. Iterative SPECT reconstruction using matched filtering for improved image quality. Nuclear Science Symposium Conference Record, 2006. IEEE 2006;4:2285-7.

13. Heller GV, Bateman TM, Cullom SJ, Hines HH, Da Silva AJ. Improved clinical performance of myocardial perfusion SPECT imaging using Astonish iterative reconstruction. Medicamundi 2009;53:43-9.
14. Case JA, Hsu BL, Bateman TM, Cullom SJ. A Bayesian iterative transmission gradient reconstruction algorithm for cardiac SPECT attenuation correction. J Nucl Cardiol 2007;14:324-33.

15. Cullom SJ, Saha K, Case JA, Hsu B, Ye J, Hines HH, et al. Accurate reconstruction of rapidly acquired 32-angle Gd- 153 scanning line source transmission projections for myocardial perfusion SPECT attenuation correction. J Nucl Cardiol. 2007;14:S98 (Abstract).

16. Cerqueira MD, Weissman NJ, Dilsizian V, Jacobs AK, Kaul S, Laskey WK, et al. Standardized myocardial segmentation and nomenclature for tomographic imaging of the heart: A statement for healthcare professionals from the Cardiac Imaging Committee of the Council on Clinical Cardiology of the American Heart Association. J Nucl Cardiol 2002;9:240-5.

17. Siegel S, Castellan NJ. Nonparametric statistics for the behavioral sciences. 2nd ed. Montreal: McGraw-Hill; 1988.

18. Newcombe RG. Two-sided confidence intervals for the single proportion: Comparison of seven methods. Stat Med 1998;17:857-72.

19. Bateman TM, Heller GV, McGhie AI, Friedman JD, Case JA, Bryngelson JR, et al. Diagnostic accuracy of rest/stress ECG-gated Rb-82 myocardial perfusion PET: Comparison with ECG-gated Tc-99m sestamibi SPECT. J Nucl Cardiol 2006;13:24-33.

20. Hendel RC, Berman DS, Cullom SJ, Follansbee W, Heller GV, Kiat H, et al. Multicenter clinical trial to evaluate the efficacy of correction for photon attenuation and scatter in SPECT myocardial perfusion imaging. Circulation 1999;99:2742-9.

21. He ZX, Iskandrian AS, Gupta NC, Verani MS. Assessing coronary artery disease with dipyridamole technetium-99m-tetrofosmin SPECT: A multicenter trial. J Nucl Med 1997;38:44-8.

22. Miller DD, Younis LT, Chaitman BR, Stratmann H. Diagnostic accuracy of dipyridamole technetium $99 \mathrm{~m}$-labeled sestamibi myocardial tomography for detection of coronary artery disease. $\mathbf{J}$ Nucl Cardiol 1997;4:18-24.

23. Links JM, DePuey EG, Taillefer R, Becker LC. Attenuation correction and gating synergistically improve the diagnostic accuracy of myocardial perfusion SPECT. J Nucl Cardiol 2002;9:183-7. 